\title{
Nueva guía de diabetes mellitus tipo 2 en Argentina
}

\author{
New guideline on type 2 diabetes mellitus in Argentina
}

\section{Comentado de:}

Argentina. Ministerio de Salud. Dirección Nacional de Abordaje Integral de Enfermedades No Transmisibles. Guía de Práctica Clínica Nacional sobre Prevención, Diagnóstico y Tratamiento de la Diabetes Mellitus Tipo 2 (DM2) $2019^{1}$

\section{Introducción}

Durante 2019 se elaboró la nueva Guía de Práctica Clínica (GPC) de Diabetes Mellitus tipo 2 en Argentina. Esta guía sur- ge del esfuerzo colaborativo del Ministerio de Salud de la Nación Argentina y de un grupo interdisciplinario de profesionales de la Dirección Nacional de Promoción de La Salud y Control de Enfermedades Crónicas No Transmisibles, siguiendo una metodología rigurosa para su elaboración, enmarcada en el enfoque GRADE para la valoración de la confianza de la evidencia y la fuerza de las recomendaciones. La guía presenta 36 recomendaciones, a continuación se resumen las recomendaciones clave (ver Tabla 1).

Tabla 1. Recomendaciones clave de la nueva guía de manejo de la diabetes mellitus tipo 2 del Ministerio de Salud de la Nación Argentina (2019). Notas: AMG: automonitoreo glucémico; DM2: diabetes mellitus tipo 2; GAA: glucemia de ayuno alterada; GPA: glucemia plasmática en ayunas; PTOG75: prueba de tolerancia oral a la glucosa.

\begin{tabular}{|c|c|}
\hline Recomendación clave & $\begin{array}{l}\text { Fuerza de recomendación } \\
\text { (confianza en la evidencia) }\end{array}$ \\
\hline $\begin{array}{l}\text { Ante alteraciones de la glucemia plasmática en ayunas (GPA), se recomienda una segunda } \\
\text { prueba para detección de casos de DM2. Si presenta: } \\
\text { - Glucemia alterada en ayunas (GAA: } 110 \text { a } 125 \mathrm{mg} / \mathrm{dl} \text { sin factores de riesgo o } 100 \text { a } 125 \mathrm{mg} / \mathrm{dl} \\
\text { con factores de riesgo para DM2): solicitar PTOG75 de forma preferencial o GPA periódicas. } \\
\text { - GPA igual o mayor de } 126 \mathrm{mg} / \mathrm{dl} \text { : repetir GPA. } \\
\text { - Glucemias discordantes (una GAA y una glucemia normal): solicitar PTOG75 de forma prefe- } \\
\text { rencial o GPA periódicas. } \\
\text { - Glucemias discordantes (una determinación de GPA igual o mayor a } 126 \mathrm{mg} / \mathrm{dl} \text { y una GPA } \\
\text { normal): repetir GPA o bien PTOG75. }\end{array}$ & $\begin{array}{l}\text { Recomendación fuerte (con- } \\
\text { fianza moderada) }\end{array}$ \\
\hline $\begin{array}{l}\text { En personas con DM2 con enfermedades en etapa terminal y expectativa de vida limitada se } \\
\text { recomienda utilizar tratamiento farmacológico solo con el objetivo de evitar hiperglucemias sin- } \\
\text { tomáticas para alcanzar un mejor balance entre los beneficios y los riesgos. }\end{array}$ & $\begin{array}{l}\text { Recomendación fuerte (con- } \\
\text { fianza moderada) }\end{array}$ \\
\hline $\begin{array}{l}\text { En personas con DM2, se recomienda iniciar tratamiento farmacológico con metformina (cuan- } \\
\text { do no presenten contraindicaciones), para lograr el control glucémico, reducir el desarrollo de } \\
\text { complicaciones cardiovasculares y probablemente reducir la mortalidad. }\end{array}$ & $\begin{array}{l}\text { Recomendación fuerte (con- } \\
\text { fianza moderada) }\end{array}$ \\
\hline $\begin{array}{l}\text { En personas con DM2 que inician tratamiento con insulina, se recomienda utilizar NPH al } \\
\text { momento de acostarse (bedtime) por ser efectiva para mejorar el control glucémico y presentar } \\
\text { un costo aceptable. }\end{array}$ & $\begin{array}{l}\text { Recomendación fuerte (con- } \\
\text { fianza moderada) }\end{array}$ \\
\hline $\begin{array}{l}\text { En personas con DM2 que reciben insulina, se recomienda el automonitoreo (AMG), individuali- } \\
\text { zando la frecuencia de control según estrategia de insulinización, objetivos de control metabólico } \\
\text { y riesgo de hipoglucemias, para guiar el tratamiento e identificar y tratar precozmente las hipo- } \\
\text { glucemias. }\end{array}$ & $\begin{array}{l}\text { Recomendación fuerte (con- } \\
\text { fianza baja) }\end{array}$ \\
\hline $\begin{array}{l}\text { En personas que al momento de diagnóstico de DM2 presenten hiperglucemia sintomática (pér- } \\
\text { dida de peso, poliuria, polifagia, polidipsia), se recomienda iniciar tratamiento con insulina al } \\
\text { momento de acostarse (bedtime) para evitar descompensaciones agudas (cetoacidosis, síndro- } \\
\text { me hiperosmolar hiperglucémico). Asociar con metformina (en ausencia de contraindicación), } \\
\text { ya que contribuye a mejorar el control glucémico y atenúa el aumento de peso, sin aumentar el } \\
\text { riesgo de hipoglucemias. }\end{array}$ & $\begin{array}{l}\text { Recomendación fuerte (reco- } \\
\text { mendación primordial) }\end{array}$ \\
\hline
\end{tabular}


En personas con DM2 que presentan hipoglucemias, se recomienda evaluar causas modificables:

Recomendación fuerte (reco-

1) adecuada ingesta de hidratos de carbono; 2) organización de la alimentación (ej. no saltear mendación primordial) comidas);

3) adecuación de tipo y horario de la actividad física;

4) sitios de aplicación de insulina (si corresponde);

y eventualmente, realizar disminución de la dosis y/o rotación del fármaco (orales y/o insulinas) para un mejor equilibrio entre los beneficios y los riesgos.

Tabla 2. Evaluación de la calidad metodológica de la guía de manejo de la diabetes mellitus tipo 2 del Ministerio de Salud de la Nación Argentina (2019)

\begin{tabular}{|c|c|}
\hline Dominio del AGREE II & Evaluación cualitativa \\
\hline Alcance y objetivo & $\begin{array}{l}\text { Los objetivos están adecuadamente descritos, claramente y fáciles de encontrar. Explica los } \\
\text { temas incluidos, excluidos y la población diana. }\end{array}$ \\
\hline Participación de los implicados & $\begin{array}{l}\text { Se especifican los nombres, especialidades/disciplinas, e instituciones en las cuales se desem- } \\
\text { peñan de los participantes en la guía, cubriendo una amplia gama de especialidades relevan- } \\
\text { tes, incluyendo metodólogos. Se ha tenido en cuenta los puntos de vista y las preferencias de } \\
\text { la población objetivo en varias instancias de la elaboración de la guía, los cuales se reflejan en } \\
\text { la formulación de las recomendaciones (las estrategias usadas se detallan en el anexo 6). No } \\
\text { se incluye evidencia local de valores y preferencias. }\end{array}$ \\
\hline Rigor en la elaboración & $\begin{array}{l}\text { Se detallan los métodos sistemáticos que se usaron para la búsqueda de evidencia, la formula- } \\
\text { ción de las recomendaciones (y su fundamentación, explicado en forma extensa en los anexos } \\
3 \text { y 4). Fue revisada externamente por dos médicas, y también hubo una instancia de revisión } \\
\text { pública; en ambos casos se describen los resultados, los cuales fueron tenidos en cuenta para } \\
\text { la modificación de las recomendaciones (Anexo 5). Aclara cuándo será actualizada y bajo qué } \\
\text { circunstancias, aunque no se mencionan los métodos a utilizar. }\end{array}$ \\
\hline Claridad en la presentación & $\begin{array}{l}\text { La información y las recomendaciones resultan precisas, concretas y detalladas, fáciles de } \\
\text { entender y de localizar en la guía. Además, hacen referencia a las modificaciones que sufrieron } \\
\text { a lo largo de la elaboración y su fundamento. }\end{array}$ \\
\hline Aplicabilidad & $\begin{array}{l}\text { Se mencionan algunas barreras a superar y se detallan varias estrategias y herramientas diri- } \\
\text { gidas al equipo de salud para implementar las distintas recomendaciones de la guía (cuestio- } \\
\text { narios, algoritmos, pruebas, cuadros de resumen, etc.). El modelo de atención de personas } \\
\text { con enfermedades crónicas, como estrategia de implementación, propone utilizar indicadores } \\
\text { de calidad de atención pero en la guía no se detalla cuáles son. Además, se tiene en cuenta } \\
\text { la accesibilidad y los costos económicos, promoviendo la intervención, programas y proyectos } \\
\text { específicos, entre otros (anexo 7). }\end{array}$ \\
\hline Independencia Editorial & $\begin{array}{l}\text { La guía fue realizada y financiada por el Ministerio de Salud de la Nación, y se declara que } \\
\text { hubo una independencia editorial. Como quienes tenían poder de decisión eran los miem- } \\
\text { bros del Panel Interdisciplinario de Consenso, fue a ellos a quienes se les hizo completar una } \\
\text { declaración de conflicto de intereses mediante un cuestionario específico. En un apartado se } \\
\text { mencionan los resultados, aunque se concluyó que el gran número de miembros del panel } \\
\text { neutralizaría los eventuales conflictos existentes (anexo 2). }\end{array}$ \\
\hline
\end{tabular}




\section{Comentario}

Tras aplicar el instrumento AGREE de valoración crítica de guías de práctica clínica (ver Tabla 2), observamos que la guía presenta una excelente calidad, con limitaciones menores en su desarrollo y su reporte. Como se anticipa en el texto de la misma, es importante la confección de diferentes versiones resumidas para facilitar su diseminación entre los profesionales de la salud, y que éstas sean lanzadas en simultáneo con la guía completa. La guía es clara, precisa, ordenada, y sus recomendaciones están adaptadas al ámbito local, con vinculación a otras guías nacionales.

En cuanto a las preferencias de los pacientes, si bien se destaca que en la elaboración de este documento se usaron datos de guías de otros países, sería interesante que para futuras actualizaciones se utilicen datos locales y se incorporen las las barreras que pueden existir en el proceso salud-enfermedad-atención (se mencionan solamente aquellas documentadas en el uso de la insulina).

La guía se enlaza con el modelo de atención de las personas con enfermedades crónicas: le da protagonismo al paciente y le otorga la posibilidad de una atención integral, con un enfoque de multimorbilidad. Sin embargo, hay aspectos no contemplados como los relacionados a la salud mental de los pacientes con diabetes, un elemento frecuentemente olvidado, pero que es considerado en otras guías como la de la Asociación Norteamericana de Diabetes (ADA, por sus iniciales en inglés), en la cual hay un apartado con recomendaciones y evidencia sobre los trastornos de ansiedad, la depresión y los trastornos de conducta alimentaria en relación a la diabetes ${ }^{2}$. Como la guía comentada está orientada a un uso en el primer nivel de atención, sería adecuado incluir estas situaciones.

En el documento se menciona el Finnish Diabetes Risk Score (FINDRISC) como un instrumento útil para identificar personas con alto riesgo de desarrollar $\mathrm{DM} 2^{3}$. Esta herramienta tiene la ventaja de que puede ser aplicada por el profesional de la salud o autoadministrado por el paciente, y contempla distintos factores de riesgo para el desarrollo de diabetes. Su puntaje tiene un rango entre 0 y 26 puntos; siendo 12 el punto de corte sugerido para realizar la medición de la glucemia como prueba de pesquisa. Todas las guías analizadas en el presente artículo lo contemplan en su desarrollo, y en la guía Argentina está en uno de los apartados para su implementación. Una calculadora digital gratuita, para uso en línea o como aplicación para teléfonos celulares, se encuentra disponible en: https://www.mdcalc.com/findrisc-finnish-diabetes-risk-score

En comparación a la Guía de Práctica Clínica de 2019 de la Asociación Latinoamericana de Diabetes (ALAD) sobre el diagnóstico, control y tratamiento de la Diabetes Mellitus tipo 2 con medicina basada en la evidencia ${ }^{4}$, la nueva guía nacional de la Argentina presenta un desarrollo metodológico más exhaustivo, concreto y específico para pacientes con DM2, orientado a la atención primaria y el manejo ambulatorio de la enfermedad. Una de sus principales diferencias radica en que no hace referencia al manejo hospitalario en descompensaciones agudas de la enfermedad, ni a pacientes con DM2 y enfermedad renal. Otra diferencia para señalar es que la guía ALAD alude a la heterogeneidad clínica de los pacientes que viven con DM2, y a las comorbilidades más frecuentes en esta población (hipertensión arterial, dislipemia), estableciendo p. ej., metas de tensión arterial, peso, perímetro abdominal, entre otras, mientras que en la guía nacional no se los menciona de manera específica. No hay discrepancias en los criterios de diagnóstico, rastreo y y en las medidas de prevención en general. La guía de la ALAD incluye otras opciones farmacológicas para su prevención (acarbosa, orlistat, rosiglitazona, agonistas del péptido tipo glucagón-1, inhibidores de la dipeptidil peptidasa-4 [IDDP-4], antagonistas del cotransportador sodio-glucosa-2 e insulina glargina) y menciona como segunda opción terapéutica a los IDDP-4, a diferencia de la guía Argentina que resalta el rol de la metformina como primera línea y el uso de varios fármacos como segunda línea, sin asignar mayor relevancia a ninguno de ellos en particular.

La Guía de 2019 sobre diabetes, prediabetes y enfermedad cardiovascular realizada por la Sociedad Europea de Cardiología y la Asociación Europea por el estudio de Diabetes ${ }^{5}$, brinda una detallada descripción en cuanto al manejo de comorbilidades vasculares que coexisten en esta población de pacientes. El concepto de prediabetes incluye a la glucemia de ayuno alterada y la intolerancia oral a la glucosa; ambas reflejan la historia natural de progresión desde la normoglucemia a la DM2. Si bien la guía argentina no utiliza este término de manera sistemática, las recomendaciones referidas a la prediabetes son homólogas entre todas las guías. Por otra parte, la guía europea establece como parte del diagnóstico inicial el dosaje de hemoglobina glicosilada (HbA1c), junto con la glucemia plasmática en ayunas y la prueba de tolerancia oral a la glucosa (PTOG 75). Por otro lado, hace alusión a los estudios que deben ser realizados luego del diagnóstico de DM2 (orina de 24 hs, fondo de ojo) para evaluar la posibilidad de daño de órgano blanco, mientras que la guía argentina no aborda esta temática y la propone para futuras actualizaciones.

Las recomendaciones de la guía de la ADA de $2020^{2}$ sobre el diagnóstico de DM2 son similares a la guía europea. En relación al tamizaje en personas asintomáticas, indica que la prueba se realice cada tres años (siempre teniendo en cuenta el FINDRISC), a diferencia de la guía Argentina que propone realizarlo cada tres a cinco años. Con el uso a largo plazo de la metformina puede observarse una deficiencia bioquímica de vitamina B12, por lo que la ADA recomienda su dosaje periódico en aquellos pacientes que se encuentren bajo este tratamiento. También se desarrollan las particularidades a tener en cuenta de acuerdo al grupo etario al que pertenece el paciente, detallando los cuidados específicos que aconsejables para los niños, los adolescentes y los adultos mayores. La guía de la ADA brinda detalles muy limitados sobre los métodos de búsqueda y análisis de la evidencia, a diferencia de la guía nacional. Sin embargo, incluye recomendaciones tipo. E similares a las que la guía argentina denomina motherhood statement (recomendación primordial) para referirse a todo lo anteriormente descrito. Consideramos que el uso del término motherhood podría evitarse, para no perpetuar simbólicamente la idea de que los cuidados están relacionados al rol de la maternidad de manera exclusiva.

Finalmente, destacamos que las tres guías coinciden que el automonitoreo glucémico se restringiría sólo a aquellos pacientes con DM2 que utilicen insulina o algún análogo de esta sustancia. 


\section{Conclusiones de los comentadores}

La nueva guía de práctica clínica nacional sobre prevención, diagnóstico y tratamiento de la DM2 de la Argentina representa un avance en el desarrollo metodológico de guías para este país, con recomendaciones claras basadas en la evidencia y herramientas para llevarlas a cabo en nuestro contexto local. Sin embargo, será necesario incluir en actualizaciones futuras mayor evidencia sobre los valores y preferencias de las personas con diabetes en nuestro país.

Tatiana Bailon [ Departamento de Salud, Universidad Nacional de La Matanza. bailontatiana5@gmail.com ]

Karen Cena [ Departamento de Salud, Universidad Nacional de La Matanza. karencena97@gmail.com ]

Sofia Rau [ Departamento de Salud, Universidad Nacional de La Matanza. sofiraus@gmail.com ]

Juan Víctor Ariel Franco [ Departamento de Salud, Universidad Nacional de La Matanza; Servicio de Medicina Familiar y Comunitaria, Hospital Italiano de Buenos Aires. juan.franco@hospitalitaliano.org.ar ]

Bailon T, Cena K, Rau S, Franco JVA Nueva guía de diabetes mellitus tipo 2 en Argentina. Evid Actual Pract Ambul. 2020;23(4):e002107. Comentado de: Argentina. Ministerio de Salud. Dirección Nacional de Abordaje Integral de Enfermedades No Transmisibles. Guía de Práctica Clínica Nacional sobre Prevención, Diagnóstico y Tratamiento de la Diabetes Mellitus Tipo 2 (DM2) 2019. Disponible en: https://bancos.salud.gob.ar/sites/default/files/202009/guia-nacional-practica-clinica-diabetes-mellitius-tipo2_2019.pdf

\section{Referencias}

1. Argentina. Ministerio de Salud. Dirección Nacional de Abordaje Integral de Enfermedades No Transmisibles. Guía de Práctica Clínica Nacional sobre Prevención, Diagnóstico y Tratamiento de la Diabetes Mellitus Tipo 2 (DM2) 2019; 2019. Available from: https://bancos.salud.gob.ar/sites/default/ files/2020-09/guia-nacional-practica-clinica-diabetes-mellitius-tipo2_2019.pdf.

2. Johnson EL, Feldman H, AButts, et al. Standards of medical care in diabetes-2020 abridged for primary care providers. Clinical Diabetes. 2020;38:10-38. Available from: 10.2337/cd20-as01.

3. Lindström J, Tuomilehto J. The diabetes risk score: A practical tool to predict type 2 diabetes risk. Diabetes Care. 2003;26(3):725-731. Available from: 10.2337/diacare.26.3.725

4. Guías ALAD sobre el Diagnóstico, Control y Tratamiento de la Diabetes Mellitus Tipo 2 con Medicina Basada en Evidencia Edición 2019. Revista ALAD. 2019;Available from: https://revistaalad.com/guias/5600AX191_guias_alad_2019.pdf.

5. Cosentino F, Grant PJ, Aboyans V, et al. 2019 ESC Guidelines on diabetes, pre-diabetes, and cardiovascular diseases developed in collaboration with the EASD. Eur Heart J. 2020;41(2):255-323. Available from: 10.1093/eurheartj/ehz486. 